TITLE:

Clear observation of the formation of nanoparticles inside the ablation bubble through a laser-induced flat transparent window by laser scattering

$\operatorname{AUTHOR}(S)$ :

Ando, Kota; Nakajima, Takashi

CITATION:

Ando, Kota ...[et al]. Clear observation of the formation of nanoparticles inside the ablation bubble through a laser-induced flat transparent window by laser scattering. Nanoscale 2020, 12(17): 9640-9646

ISSUE DATE:

2020-05-07

URL:

http://hdl.handle.net/2433/252374

RIGHT:

This is the accepted manuscript of the article, which has been published in final form at

https://doi.org/10.1039/c9nr10560c.; The full-text file will be made open to the public on 7 May 2021 in accordance with publisher's 'Terms and Conditions for Self-Archiving'.; この論文は出版社版でありません。引用の際には出版社版をご 確認じ利用ください。; This is not the published version. Please cite only the published version. 


\title{
ARTICLE
}

\section{Clear observation of the formation of nanoparticles inside the ablation bubble through a laser-induced flat transparent window by laser scattering}

Received 00th January 20xx, Accepted 00th January 20xx DOI: $10.1039 / x 0 x \times 00000 x$

\begin{abstract}
Kota Ando a and Takashi Nakajima *a
To understand the formation mechanism of nanoparticles via laser ablation in liquids direct observation of the dynamics inside the ablation bubble is essential. One powerful technique for this is small-angle $x$-ray scattering. Applying the laserscattering technique in a similar context faces some difficulties, because, firstly the probe laser does not easily go into the bubble due to the curved bubble surface, and secondly the extremely weak scattering signal from inside the bubble is overwhelmed by the very strong reflection at the bubble surface. In this paper we solve those two problems by sending the probe laser into the bubble through a laser-induced flat transparent window, and demonstrate the clean observation of laser-scattering signals from the inside of the ablation bubble. We observe the signature of the formation of nanoparticles around the two areas inside the bubble, i.e., around the central area and apex inside the bubble. The scattering signals originating from the centre of the bubble persist throughout the entire growth and shrinkage stages of the bubble, while practically no scattering signals are found outside the bubble and this clearly implies that almost all nanoparticles are formed inside the bubble. Interestingly, the scattering signals originating from the apex inside the bubble gradually emerges after the bubble reaches the maximum size, and they become brighter as it further shrinks. Those findings are consistent with the scenario of nanoparticle formation obtained by small-angle x-ray scattering experiments.
\end{abstract}

\section{Introduction}

Irradiation of a focused high intensity laser pulse onto a solid target in vacuum/gases/liquids results in the nonlinear photoabsorption into the target material, and this triggers laser ablation. It is a simple and very effective technique to remove material from the target, and very unusual dynamics can appear if ultrafast bursts of laser pulses are applied to the target. ${ }^{1}$ One of the useful applications of laser ablation in liquids (LAL) is a synthesis of nanomaterials. ${ }^{2-5}$ The main concern of nanomaterial synthesis by $\mathrm{LAL}$ is a particle size and its distribution, since the small particle size helps to increase the efficiency of application in many cases. Toward this goal deep understanding of the entire LAL processes is essential. However, that is not easy, because the surrounding liquid plays an important role in the following processes upon ablation: (1) Formation of laser-induced plasma, (2) formation, expansion, and then shrinkage of the ablation bubble which consists of the vapour of the surrounding liquid as well as the fractions of target material in vapour, liquid, and solid phases, and (3) eventually the release of nanoparticles and their agglomerates into the bulk liquid. Therefore, in spite of the models proposed

\footnotetext{
a. Institute of Advanced Energy, Kyoto University, Gokasho, Uji, Kyoto 611-0011, Japan.E-mail:nakajima@iae-kyoto-u.ac.jp

+ Footnotes relating to the title and/or authors should appear here.

Electronic Supplementary Information (ESI) available: Supplementary movie and figures are available. See DOI: 10.1039/x0xx00000x
}

in the literature ${ }^{6}$ the formation mechanism of nanoparticles by LAL is still an intriguing and not fully answered question.

There are a few known experimental techniques to investigate the ablation bubble dynamics. The most commonly used one is the time-resolved shadowgraphy, ${ }^{7-9}$ but it can only measure the time-varying shape of the bubble. For this reason the shadowgraphy is often employed with other techniques such as plasma spectroscopy, ${ }^{10-13}$ optical beam deflection, ${ }^{14,15}$ light scattering, ${ }^{16-19}$ and $x$-ray scattering. ${ }^{20-23}$ The use of highspeed camera also helps a lot to track the bubble evolution after the single ablation event. ${ }^{24-26}$ To obtain information on the size and mass of particles inside the bubble small-angle $x$-ray scattering (SAXS) has been proved to be very powerful, ${ }^{20-23}$ and Plech and his co-workers have demonstrated that the combined use of SAXS with wide-angle x-ray scattering (WAXS) can provide information on not only the size and mass inside the bubble but also their crystallinity. ${ }^{27}$

Other than the nanomaterial synthesis, laser ablation can be used for the spectroscopic trace analysis, and this specific branch is called laser-induced breakdown spectroscopy (LIBS). ${ }^{28-30}$ Along this line the ablation bubble dynamics are also being investigated by using a single long pulse, ${ }^{11,12,31,32}$ double pulses, ${ }^{11,33-36}$ multi-pulse, ${ }^{37}$ etc.

In contrast to the significant recent advances using the x-rayscattering-based technique mentioned above, the lightscattering-based technique seems to be left behind. Although some of the papers in the literature have reported the use of light-scattering to observe the dynamics inside the ablation bubble, ${ }^{16-19}$ distinction of the very weak and meaningful 


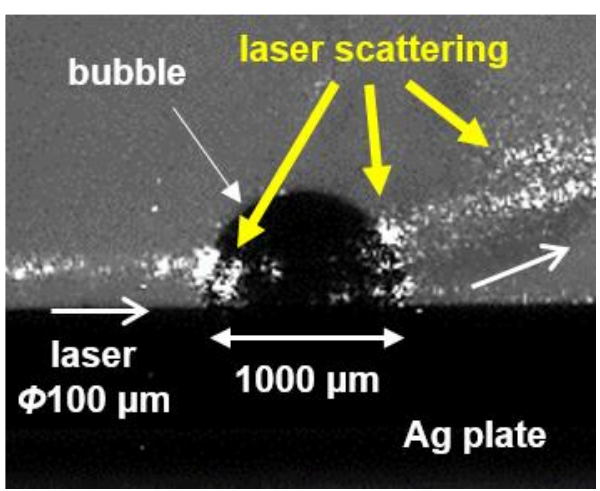

Fig. 1 Example of the laser scattering image superposed on the shadowgraph image under the conventional light-scattering setup where the pump laser is at normal incidence to the Ag target for ablation while the probe laser goes parallel to the target surface. Although the diameter of the probe laser is as small as 100 $\mu \mathrm{m}$ which is far smaller than that of the bubble, $1000 \mu \mathrm{m}$, the above image shows the very strong light scattering at the front and back surfaces of the bubble. What we see inside the bubble is a real one or an artifact is not clear. Note that the scattering signals are also seen along the probe beam line before and after it goes through the bubble, since we have already shot a few pump laser pulses to adjust the optical alignment of the pump laser, probe laser, and imaging camera, and hence there are already many nanoparticles outside the bubble which serve as scatterers.

scattering signals from the undesired and far dominant scattering signals at the bubble surface is extremely difficult, and actually there would be no clear distinction between them. To clarify the point, we present in Fig. 1 an example of the laser scattering image we have obtained under the conventional light-scattering setup: The pump laser is focused onto the $\mathrm{Ag}$ target in water for ablation with normal incidence, and the probe laser with a diameter much smaller than that of the bubble is sent parallel to the target surface. Under this geometry, even after the precise adjustment of pump laser, probe laser, and imaging camera, the very strong scattering at the bubble surface is unavoidable. If the probe beam diameter is comparable or larger than that of the bubble, it would be even worse. Thus, by comparing Fig. 1 of this paper with Fig. 2 of ref.16, for example, we cannot confidently claim that we have certainly observed the inside of the bubble by light scattering under the conventional setup, as correctly pointed out in ref.17,18,22. This problem mainly arises from the large curvature of the bubble surface with respect to the incident probe beam diameter. More precise adjustment of optical components and the use of the probe beam with even smaller diameter may partially solve the problem, but there is no perfect solution, because there is a small shot-to-shot fluctuation of the bubble position on the target, and more importantly, the bubble size and hence the surface curvature changes in time.

The purpose of this paper is to propose a new lightscattering-based technique and demonstrate the clear observation of light scattering from inside the bubble. As shown in Fig. 2(b), the point of our technique is to introduce the probe laser into the ablation bubble through a flat and transparent window created by the pump laser for ablation of a thin Ag film on a glass substrate. As we will show later on in this paper, our setup allows us to effectively suppress the light scattering from the bubble surface. From the simple inverse light scattering analysis we find that, roughly speaking, the scattering signals originate from the two areas in the bubble, i.e., around the apex and the central area of the bubble. The scattering signals from the former clearly show the dependence on the polarization of the probe laser, which is a good evidence that the scatterers located near the apex inside the bubble are spherical nanoparticles. In contrast, the latter, although the signals are extensively distributed around the centre of the bubble, hardly shows such a dependence, which implies the occurrence of multiple scatterings due to the high number density of the nanoparticles around the central area in the bubble. Our findings are qualitatively consistent with the scenario based on the experimental observation with SAXS. ${ }^{21,22,27}$

\section{Experimental}

\section{Preparation of Ag films}

In this experiment, we employ a magnetron sputtering device (MSP-mini, Vacuum Device Ltd.) with an Ag target (purity 99.98 $\%$, Nilaco) to deposit an Ag film on a borosilicate glass substrate $(18 \times 18 \times 0.15 \mathrm{~mm}$, Matsunami Ltd.) as a laser ablation target. After trial and error for the Ag film thickness we find that the thickness of $\sim 100 \mathrm{~nm}$ (measured by atomic force microscope (VN-8000, Keyence)) is appropriate to make a nearly transparent window upon ablation without damaging the glass substrate.

\section{Laser ablation}

Fig. 2 shows the experimental setup. The second harmonic (532 $\mathrm{nm}$ ) of the Q-switched Nd:YAG laser (GAIA2, Rayture Systems Co., Ltd., pulse duration $5 \mathrm{~ns}$, max pulse energy $60 \mathrm{~mJ}$ at 1064 $\mathrm{nm}, \mathrm{M}^{2 \sim 15}$ ) is employed for the pump laser. The pump laser beam is guided into an acrylic cuvette $(50 \mathrm{~mm} \times 50 \mathrm{~mm} \times 50$ $\mathrm{mm}$ ) (Fig. 2a) which is mounted on an XYZ-stage, and focused onto the $\mathrm{Ag}$ film (with the film side up) immersed in deionized water (Fig. 2b). As mentioned before, the Ag film thickness, $\sim 100 \mathrm{~nm}$, and the pump laser fluence at the Ag film, $\sim 3 \mathrm{~J} / \mathrm{cm}^{2}$ (estimated from the pulse energy, $3.7 \pm 0.1 \mathrm{~mJ}$, and the focused pump beam diameter at the $\mathrm{Ag}$ film, $\sim 400 \mu \mathrm{m}$ ) which may be about $10 \%$ off, are carefully chosen so that the diameter of the ablated hole on the Ag film (Fig. 2c) becomes the appropriate size, $\sim 400 \mu \mathrm{m}$, through which the probe laser is sent into the ablation bubble. After every pump pulse the acrylic cuvette is translated by $1 \mathrm{~mm}$ with a stepping motor so that a fresh film is ablated. Note that the incident angle and spatial profile of the pump laser are also very important to create the round ablation hole for the homogeneous probe laser illumination inside the bubble (Supplementary Fig. S1+). To ensure that ablation of the glass substrate does not occur at this pump laser fluence we irradiate the Ag film by several pump pulses without moving the irradiating position, and find that the spatial profile of the transmitted probe laser is not distorted. To perform the scattering experiments in clean water we employ the protocol described in Supplementary Fig. S2+.

\section{Shadowgraph and laser-scattering}


(a)

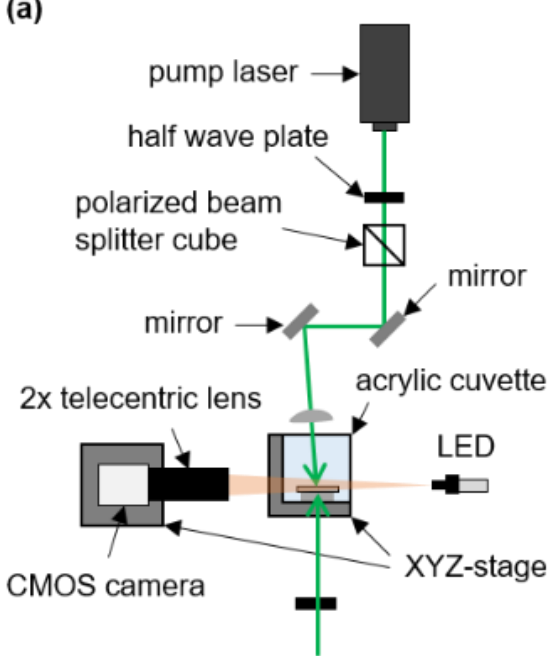

(b)

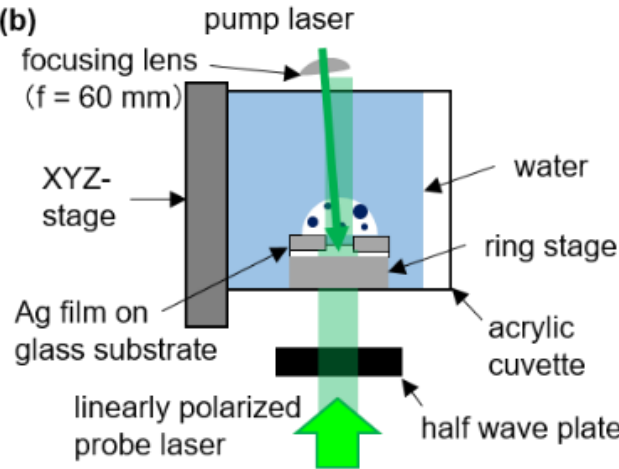

(c)

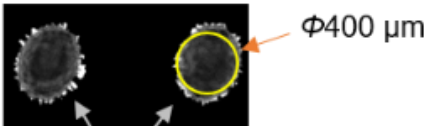

ablated holes on Ag film

Fig. 2 (a) Top view of the entire experimental setup. (b) Side view of the acrylic cuvette and the probe laser which is depicted from the side of the CMOS camera. (c) Optical images of the ablated holes on the Ag film created by the single pump laser pulses.

Within a few $\mu$ s after the pump pulse, an ablation bubble starts to grow. We employ the second harmonic of another Qswitched Nd:YAG microlaser (STA-01-07-AQ, Standa Ltd., pulse duration $0.8 \mathrm{~ns}$, max pulse energy $100 \mu \mathrm{J}$ at $1064 \mathrm{~nm}, \mathrm{M}^{2 \sim 1.2)}$ as a probe laser, and send it from the side of the cuvette through the flat transparent window created by the pump laser (Fig. 2b). To purify and control the polarization direction of the probe laser a Glan-laser calcite polarizer (Thorlabs, Inc.) and a zero-order half wave plate (Thorlabs, Inc.) are placed before the probe laser enters the cuvette. The probe beam diameter is adjusted to be $\sim 0.8 \mathrm{~mm}$, which is sufficiently larger than that of the transparent window created on the Ag film (Fig. 2c), for the secure entry into the ablation bubble. The probe laser fluence is set to $1.8 \mathrm{~mJ} / \mathrm{cm}^{2}$ which is about $1 / 1700$ of that for the pump laser, and we confirm that ablation does not occur by the probe pulse itself. Scattering signals by the objects inside the bubble are collected with a telecentric objective lens (KCM-2T, Tokina,

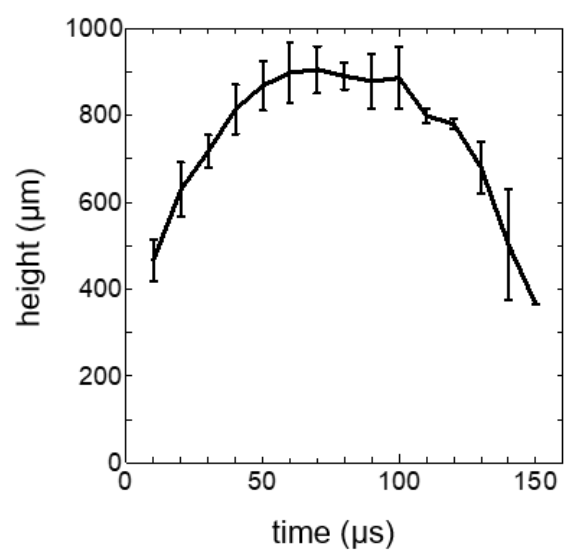

Fig. 3 Temporal change of the bubble height after the pump laser pulse. The bubble height is defined by the distance between the apex of ablation bubble and the glass substrate. Note that this graph is for bubble height before the first collapse of the bubble at $\sim 160 \mu \mathrm{s}$. magnification 2.0x, working distance $65 \mathrm{~mm}$, numerical aperture 0.038), and imaged onto the two-dimensional sensor of a CMOS camera (VCXU-04, Baumer, maximum frame rate 430 $\mathrm{fps}$, minimum exposure time $1 \mu \mathrm{s})$, which is also mounted on another XYZ-stage, as shown in Fig. 2a. The details of the experimental arrangement are described in Supplementary Fig. $\mathrm{S} 2+$. The same objective lens and camera are also used to simultaneously take the shadowgraph of the bubble under the LED illumination (AS3000, As One Co.). The pump laser, probe laser, and CMOS camera are synchronized through the delay pulse generator (DG645, Stanford Research Systems). To avoid the motion blur of the rapidly expanding/shrinking bubble the exposure time of the camera is set to $2 \mu \mathrm{s}$. By varying the delay time between the pump and probe lasers together with the exposure timing of the camera in the range of 10-180 $\mu$ s with a $10 \mu$ step, we can obtain a series of laser scattering images superposed on the shadowgraph images of the bubble. Fig. 3 shows the temporal change of the bubble height estimated from the shadowgraph images taken at different delay times after the pump laser. At $60 \mu$ s after the pump laser the bubble height reaches the maximum, and then, it begins to shrink and eventually collapses at $\sim 160 \mu \mathrm{s}$. After the first collapse the rebound of the bubble is observed at 170-180 $\mu$ s with a completely distorted bubble shape (not shown here).

\section{Results and discussion}

Fig. 4 shows the sequential snapshots of the laser scattering images superimposed on the shadowgraph images at different delay times after the pump pulses with two differently polarized probe pulses, respectively. The movie of laser scattering images is shown in Supplementary Movie S1t. While the laser scattering images by two differently polarized probe pulses are very similar up to the delay of $<130 \mu \mathrm{s}$, some differences start to emerge after that. Namely, up to the delay of $<130 \mu$ s we can see the bright area just above the transparent window together 


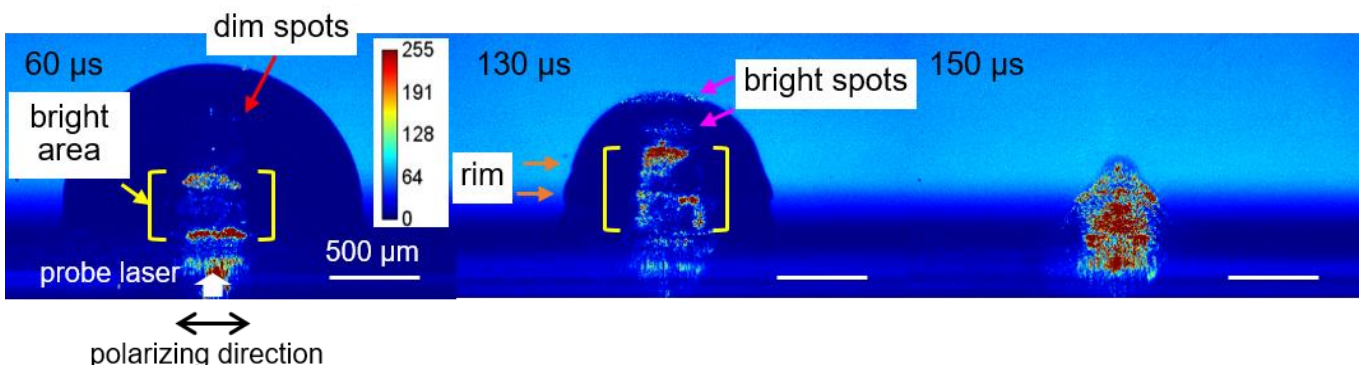

polarizing direction

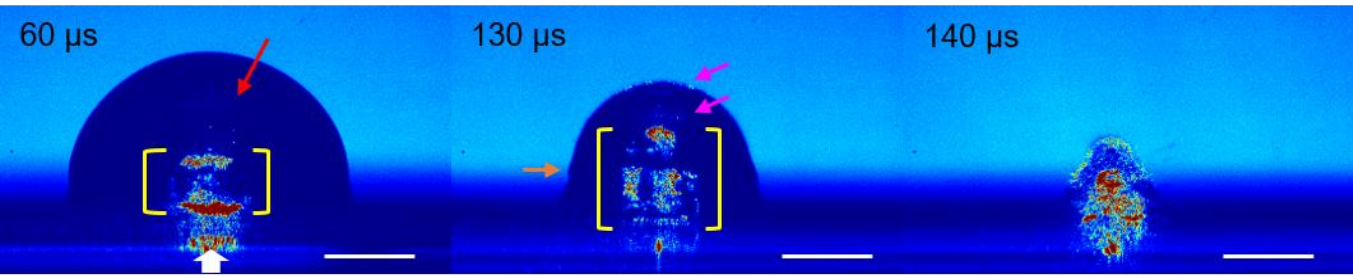

$\otimes$

Fig. 4 Laser scattering images superposed on the shadowgraph images at different delays after the pump pulse. The probe laser is (top) horizontally and (bottom) verticallyto-paper polarized, as indicated by the arrow and cross mark.

with many dim spots over it, as indicated in Fig. 4. Interestingly, they are all inside the ablation bubble and along the probe laser path, and appear at nearly the same positions for all delays. Besides, we hardly see any bright spots outside the bubble, and this is totally different from those reported in ref.16 where the scattered laser light by the bubble surface and the nanoparticles in the bubble seem to have been detected without distinction. ${ }^{16}$ Knowing the advantage of our laser scattering setup over the conventional one we can say that the dim spots we see in Fig. 4 before $130 \mu$ s already originate from Ag nanoparticles inside the bubble, since the brightness of the dim spots seems to depend on the polarization direction of the probe laser, i.e., the dim spots by the horizontally polarized probe pulse look a little brighter than those by the vertically polarized one. These observations are consistent with the Rayleigh scattering theory. ${ }^{38}$ Strictly speaking, the Rayleigh scattering theory is applicable for the scattering object whose diameter, $d$, is much smaller than the wavelength, $\lambda$, of the incident light, i.e., $\pi d \ll$ $\lambda$, but the polarization dependence of scattering intensity is approximately valid up to $\pi d \approx \lambda$. An example is shown in Supplementary Fig. S3+ for Ag nanoparticles (diameter $100 \mathrm{~nm}$ ) dispersed in water. Therefore, the origin of the dim spots is likely to be Ag nanoparticles with a diameter of a few hundred $\mathrm{nm}$. Now, we turn to the bright area indicated in Fig. 4. Before the bubble reaches the maximum size, say, at $60 \mu \mathrm{s}$ in Fig. 4, the brightness of the bright area by the horizontally and vertically polarized probe pulses are very similar, and this is perhaps because multiple scatterings are taking place due to the high number density of scatterers around the central area in the bubble. At the delay of $\geq 130 \mu \mathrm{s}$, however, the laser scattering images from inside the bubble show a much clearer dependence on the probe laser polarization (Fig. 4). As indicated by pink arrows for the panels of $130 \mu \mathrm{s}$ in Fig. 4 we find a few bright spots not only above the bright area but also around the apex of the bubble when the probe pulse is horizontally polarized, while darker spots appear at the similar positions when the probe pulse is vertically-to-paper polarized. As mentioned above, this is a clear evidence that these bright spots correspond to the laser scattering images of $\mathrm{Ag}$ nanoparticles. The intensity and number density of these bright spots rapidly increase in the time rage of $110-140 \mu \mathrm{s}$ (Supplementary Movie $\mathrm{S}^{+}{ }^{+}$). We also find that these spots are

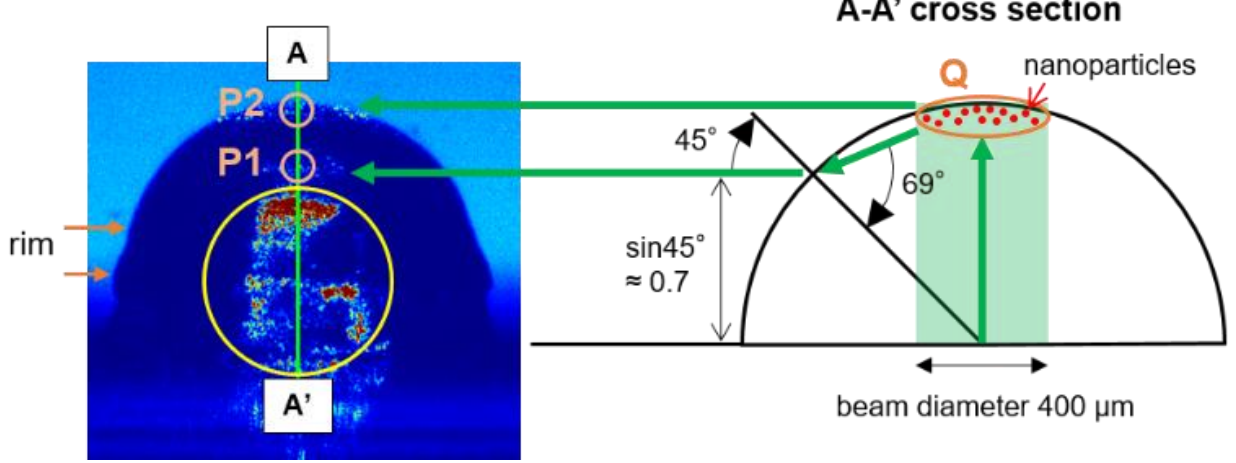

Fig. 5 (Left) Laser scattering image superimposed on the shadowgraph image by the horizontally polarized probe pulse at the delay of $130 \mu$ s. (Right) Optical path of the scattering light cut at A-A' cross section. 
all located inside the rapidly shrinking bubble, and never appear outside the bubble.

Having commented on the general features of the laser scattering images inside the bubble we try to figure out from where in the bubble the scattering light comes from. This is an inverse light scattering problem, and the scattering signals we consider below are those at points $\mathrm{P} 1, \mathrm{P} 2$, and the broad bright area circled by yellow line in the left panel of Fig. 5 . Recall that the bubble in water serves as a concave lens, and the numerical aperture of the employed objective lens is small so that the scattering light from inside the bubble goes parallel to the optical axis toward the CMOS camera. For simplicity we assume that the bubble is a perfect hemisphere with the refractive indices of bubble and water at the probe laser wavelength (532 $\mathrm{nm}$ ) being 1.0 and $1.32,{ }^{39}$ respectively, and to start with, geometric-optically solve the inverse light scattering problem for the scattering signals from points $\mathrm{P} 1$ and $\mathrm{P} 2$, as marked in the left panel of Fig. 5. The solved rays of the scattered light as well as incident probe pulse are depicted by green arrows in the right panel of Fig. 5. Since the relative height of point $P 1$ with respect to the apex of the bubble is 0.7 , the incident angle of inverse light from point $\mathrm{P} 1$, which propagates opposite to the direction of the green arrow, is $\sin ^{-1} 0.7^{\sim} 45^{\circ}$. Then, the refraction angle is calculated to be $\sin ^{-1}\left(1.32 \sin 45^{\circ}\right) \sim 69^{\circ}$ by Snell's law. Therefore, we find that the scattering signal from point $P 1$ originates from the scatterers located at point $Q$ in the right panel of Fig. 5. Analysis of the scattering signal from point P2 is much easier, and it can be understood as scattering light that has reached the camera directly from point $Q$ without refraction (left horizontal green arrow from point $Q$ in the right panel of Fig.5). As a result, we can say that the bright spots around points $\mathrm{P} 1$ and $\mathrm{P} 2$ both originate from the scatterers, which are presumably $\mathrm{Ag}$ nanoparticles as explained above, located around the apex inside the bubble, i.e., around point $Q$. According to this geometric-optical analysis, the highest possible position of the scatterers on line $A-A^{\prime}$ for the associated scattering signals to reach the camera after refraction is point $\mathrm{P} 1$, since point $\mathrm{P} 1$ corresponds to the highest possible position inside the bubble, i.e., point $Q$. Similar precise analysis for the bright scattering signals circled by yellow line in the left panel of Fig. 5 is not possible due to the obvious distortion of the bubble shape (i.e., presence of the rims on the bubble surface as indicated in the left panel of Fig. 5) which extends from the foot to the middle height of the bubble. We suspect that such a wavy structure on the bubble surface works as a sort of lens to result in the stripey bright pattern of the scattering image, as circled by yellow line in the left panel of Fig. 5 . Without resorting to the precise inverse light scattering analysis, however, it is clear that those signals originate from the high number density scatterers located around the central area in the bubble. We note that the influence of LED light on the scattering images is subtle and may be neglected for the analysis of scattering images (see Supplementary Fig. $\mathrm{S}^{+}+$and Fig. $\mathrm{S} 5+$ ).

Now, we discuss the possible correlation between the bubble dynamics and nanoparticle growth/aggregation. According to the SAXS measurements of nanoparticles in the ablation bubble, ${ }^{40}$ the authors have found that the bubble interface constitutes a strong boundary for the nanoparticles and the majority of particle mass is confined to the region close to the ablation target surface when the bubble collapses. The locations of the scatterers (nanoparticles) we have found through the laser scattering technique are around the central area and apex and in the bubble, and these findings are consistent with the scenario of nanoparticle formation based on the $\mathrm{X}$-ray-based technique. ${ }^{40}$ The particle size estimated by $\mathrm{X}$ ray diffraction is reported to be in the range of $30-70 \mathrm{~nm} \cdot{ }^{21,22,27}$ In laser scattering experiments, the particle size cannot be estimated by the measurement at a single scattering angle. The best we can say about the particle size is that, by referring to the intensity of the measured scattering signals for the case of $\mathrm{Ag}$ nanoparticles with a diameter of $100 \mathrm{~nm}$ (Supplementary Fig. $\mathrm{S3}^{+}$) which is relatively easy to detect, the detection limit of our system for isolated nanoparticles would be around $\sim 70 \mathrm{~nm}$ for which the signal intensity becomes about $10 \%$ of that for a $100 \mathrm{~nm}$ nanoparticle. The size of nanoparticles around the central area of the bubble can be smaller than this, since the number density is presumably very high, which works positively for the detection. In spite of its weakness to estimate the particle size, the main advantage of laser scattering technique is that we can obtain the overall information on the spatial distribution of nanoparticles inside the bubble, since the scattering image of the entire bubble can be obtained by a single probe pulse at a given delay.

\section{Conclusions and outlook}

We have developed a new technique to clearly observe the inside of the ablation bubble by light scattering. The conventional light-scattering setup for the ablation bubble, i.e., sending the probe laser from the side of the curved bubble surface, does not work well, since most of the scattering signal comes from the undesired and unavoidable reflection at the bubble surface. To solve this long-standing problem, we have employed a magnetron-sputtered thin $\mathrm{Ag}$ film on a glass substrate as an ablation target, and adjust the film thickness and pump laser parameters so that only the film is ablated without damaging the glass substrate.

Upon ablation a flat transparent window with a diameter of a few hundred $\mu \mathrm{m}$ is created on the Ag film, through which we can securely send the linearly polarized probe laser to illuminate the objects inside the bubble. Combined with the conventional shadowgraph setup we can take the laser scattering images superposed on the shadowgraph images at different time delays after the pump pulse. Once the laser scattering images have been obtained from the inside of the bubble, we can geometric-optically solve the inverse light scattering problem to figure out from where inside the bubble the scattering signals come from. We have found that the scattering signals originate from the two areas in the bubble, i.e., around the central area and apex inside the bubble. The scattering signals originating from the centre of the bubble are the dominant ones, and they persist throughout the entire growth and then shrinkage stages of the bubble, while those originating from the apex inside the bubble gradually emerges 
after the bubble reaches the maximum size, and they become brighter as it further shrinks. Interestingly, practically no scattering signals are found outside the bubble, and this clearly implies that almost all nanoparticles are formed inside the bubble. Regardless of the limitation of the technique we have developed in this paper, the information we can obtain through this table-top laser-based technique would be complementary to that by the $\mathrm{x}$-ray-based technique, and the main advantage of the laser-based technique in the context of nanoparticle formation inside the ablation bubble is that we can obtain the scattering image of the entire bubble by a single probe pulse at a given delay after the pump pulse. Therefore, it can serve as a convenient workhorse to deepen the understanding of ablation bubble dynamics for the efficient nanomaterial synthesis. The light scattering experiments described in this work can be further improved by employing different colours for the probe laser, and that is our future project.

\section{Conflicts of interest}

There are no conflicts to declare.

\section{Acknowledgements}

This work was supported by the Collaboration Program of the Laboratory for Complex Energy Processes, Institute of Advanced Energy, Kyoto University.

\section{References}

1 C. Kerse, H. Kalaycloĝ Lu, P. Elahi, B. Çetin, D. K. Kesim, Ö. Akçaalan, S. Yavaş, M. D. Aşlk, B. Öktem, H. Hoogland, R. Holzwarth and F. Ö. Ilday, Nature, 2016, 537, 84-88.

2 H. Zeng, X. W. Du, S. C. Singh, S. A. Kulinich, S. Yang, J. He and W. Cai, Adv. Funct. Mater., 2012, 22, 1333-1353.

3 V. Amendola and M. Meneghetti, Phys. Chem. Chem. Phys. 2013, 15, 3027-3046

4 J. Xiao, P. Liu, C. X. Wang and G. W. Yang, Prog. Mater. Sci., 2017, 87, 140-220.

5 D. Zhang, B. Gökce and S. Barcikowski, Chem. Rev., 2017, 117, 3990-4103.

6 T. E. Itina, J. Phys. Chem. C, 2011, 115, 5044-5048.

7 T. Tsuji, Y. Tsuboi, N. Kitamura and M. Tsuji, Appl. Surf. Sci., 2004, 229, 365-371.

8 V. Lazic, S. Jovicevic and M. Carpanese, Appl. Phys. Lett., 2012, 101, 054101.

9 A. De Bonis, M. Sansone, L. D'Alessio, A. Galasso, A. Santagata and R. Teghil, J. Phys. D. Appl. Phys., 2013, 46, 445301.

10 T. Tsuji, Y. Okazaki, Y. Tsuboi and M. Tsuji, Japanese J. Appl. Physics, Part 1 Regul. Pap. Short Notes Rev. Pap., 2007, 46, 1533-1535.

11 T. Sakka, A. Tamura, T. Nakajima, K. Fukami and Y. H. Ogata, J. Chem. Phys., 2012, 136, 1-5.

12 B. Thornton, T. Sakka, T. Masamura, A. Tamura, T. Takahashi and A. Matsumoto, Spectrochim. Acta-Part B At. Spectrosc., 2014, 97, 7-12.

13 A. Tamura, A. Matsumoto, T. Nakajima, K. Fukami, Y. H. Ogata, N. Nishi and T. Sakka, J. Appl. Phys., 2015, 117, 023302.

14 R. Petkovšek and P. Gregorčič, J. Appl. Phys., 2007, 102, 044909.
15 J. Chen, X. Li, Y. Gu, H. Wang, X. Song and H. Zeng, J. Colloid Interface Sci., 2017, 489, 38-46.

16 W. Soliman, N. Takada and K. Sasaki, Appl. Phys. Express, 2010, 3, 035201.

17 A. De Giacomo, M. Dell'Aglio, A. Santagata, R. Gaudiuso, O. De Pascale, P. Wagener, G. C. Messina, G. Compagnini and S. Barcikowski, Phys. Chem. Chem. Phys., 2013, 15, 3083-3092.

18 M. Dell'Aglio, R. Gaudiuso, O. De Pascale and A. De Giacomo, Appl. Surf. Sci., 2015, 348, 4-9.

19 M. Takeuchi and K. Sasaki, Appl. Phys. A Mater. Sci. Process., 2016, 122, 312.

20 L. Lavisse, J. L. Le Garrec, L. Hallo, J. M. Jouvard, S. Carles, J. Perez, J. B. A. Mitchell, J. Decloux, M. Girault, V. Potin, H. Andrzejewski, M. C. Marco De Lucas and S. Bourgeois, Appl. Phys. Lett., 2012, 100, 164103.

21 S. Ibrahimkutty, P. Wagener, A. Menzel, A. Plech and S. Barcikowski, Appl. Phys. Lett., 2012, 101, 103104.

22 P. Wagener, S. Ibrahimkutty, A. Menzel, A. Plech and S Barcikowski, Phys. Chem. Chem. Phys., 2013, 15, 3068-3074.

23 S. Ibrahimkutty, P. Wagener, T. D. S. Rolo, D. Karpov, A. Menzel, T. Baumbach, S. Barcikowski and A. Plech, Sci. Rep., 2015, 5, 16313.

24 R. Tanabe, T. T. P. Nguyen, T. Sugiura and Y. Ito, Appl. Surf. Sci., 2015, 351, 327-331.

25 J. Lam, J. Lombard, C. Dujardin, G. Ledoux, S. Merabia and D. Amans, Appl. Phys. Lett., 2016, 108, 074104.

26 C. Y. Shih, R. Streubel, J. Heberle, A. Letzel, M. V. Shugaev, C. Wu, M. Schmidt, B. Gökce, S. Barcikowski and L. V. Zhigilei, Nanoscale, 2018, 10, 6900-6910.

27 S. Reich, A. Letzel, A. Menzel, N. Kretzschmar, B. Gökce, S. Barcikowski and A. Plech, Nanoscale, 2019, 11, 6962-6969.

28 T. Lippert and J. T. Dickinson, Chem. Rev., 2003, 103, 453-485.

29 D. W. Hahn and N. Omenetto, Appl. Spectrosc., 2010, 64, 335366.

30 F. J. Fortes, J. Moros, P. Lucena, L. M. Cabalín and J. J. Laserna, Anal. Chem., 2013, 85, 640-669.

31 T. Sakka, H. Oguchi, S. Masai, K. Hirata, Y. H. Ogata, M. Saeki and H. Ohba, Appl. Phys. Lett., 2006, 88, 061120.

32 A. Matsumoto, A. Tamura, A. Kawasaki, T. Honda, P. Gregorčič, N. Nishi, K. I. Amano, K. Fukami and T. Sakka, Appl. Phys. A Mater. Sci. Process., 2016, 122, 234.

33 C. Gautier, P. Fichet, D. Menut, J. L. Lacour, D. L'Hermite and J. Dubessy, Spectrochim. Acta-Part B At. Spectrosc., 2004, 59, 975-986.

34 C. Gautier, P. Fichet, D. Menut and J. Dubessy, Spectrochim. Acta - Part B At. Spectrosc., 2006, 61, 210-219.

35 V. I. Babushok, F. C. DeLucia, J. L. Gottfried, C. A. Munson and A. W. Miziolek, Spectrochim. Acta-Part B At. Spectrosc., 2006, 61, 999-1014.

36 A. De Giacomo, M. Dell'Aglio, O. De Pascale and M. Capitelli, Spectrochim. Acta - Part B At. Spectrosc., 2007, 62, 721-738.

37 A. Tamura, T. Sakka, K. Fukami and Y. H. Ogata, Appl. Phys. A Mater. Sci. Process., 2013, 112, 209-213.

38 M. B. and E. Wolf, Principles of Optics 7th Ed., Cambridge University Press, 1999.

39 P. Schiebener, J. Straub, J. M. H. Levelt Sengers and J. S Gallagher, J. Phys. Chem. Ref. Data, 1990, 19, 677-717.

40 S. Reich, P. Schönfeld, P. Wagener, A. Letzel, S. Ibrahimkutty, B. Gökce, S. Barcikowski, A. Menzel, T. dos Santos Rolo and A. Plech, J. Colloid Interface Sci., 2017, 489, 106-113.

41 P. Laven, A computer program for scattering of light from a sphere using Mie theory \& the Debye series, http://www.philiplaven.com/mieplot.htm, (accessed September 20, 2019). 


\title{
Supplementary Information for
}

Clear observation of the formation of nanoparticles inside the ablation bubble through a laser-induced flat transparent window by laser scattering

\author{
Kota Ando ${ }^{\mathrm{a}}$ and Takashi Nakajima ${ }^{\mathrm{a}^{*}}$
}

anstitute of Advanced Energy, Kyoto University, Gokasho, Uji, Kyoto 611-0011, Japan

*Corresponding author: nakajima@iae.kyoto-u.ac.jp 
(a)
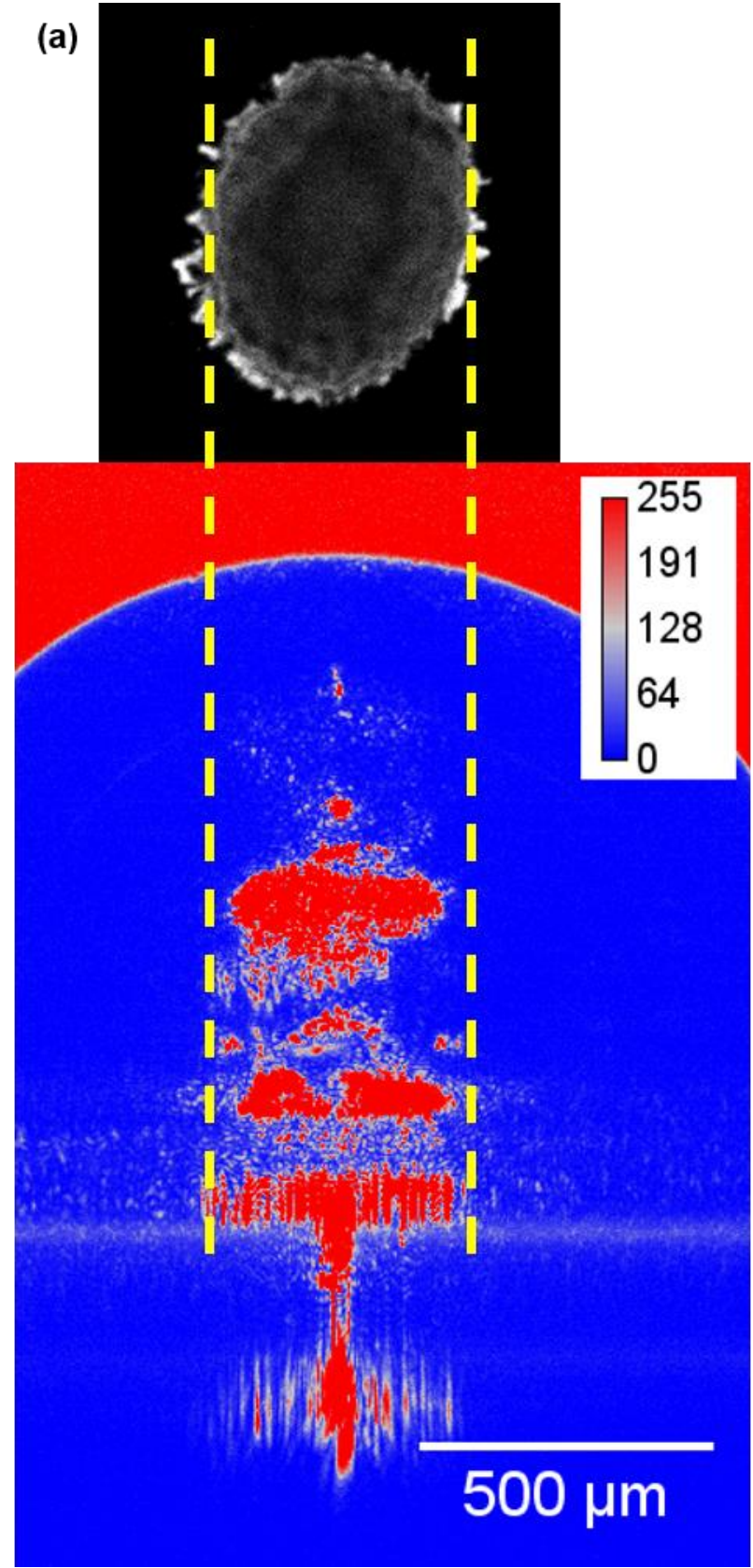

(b)

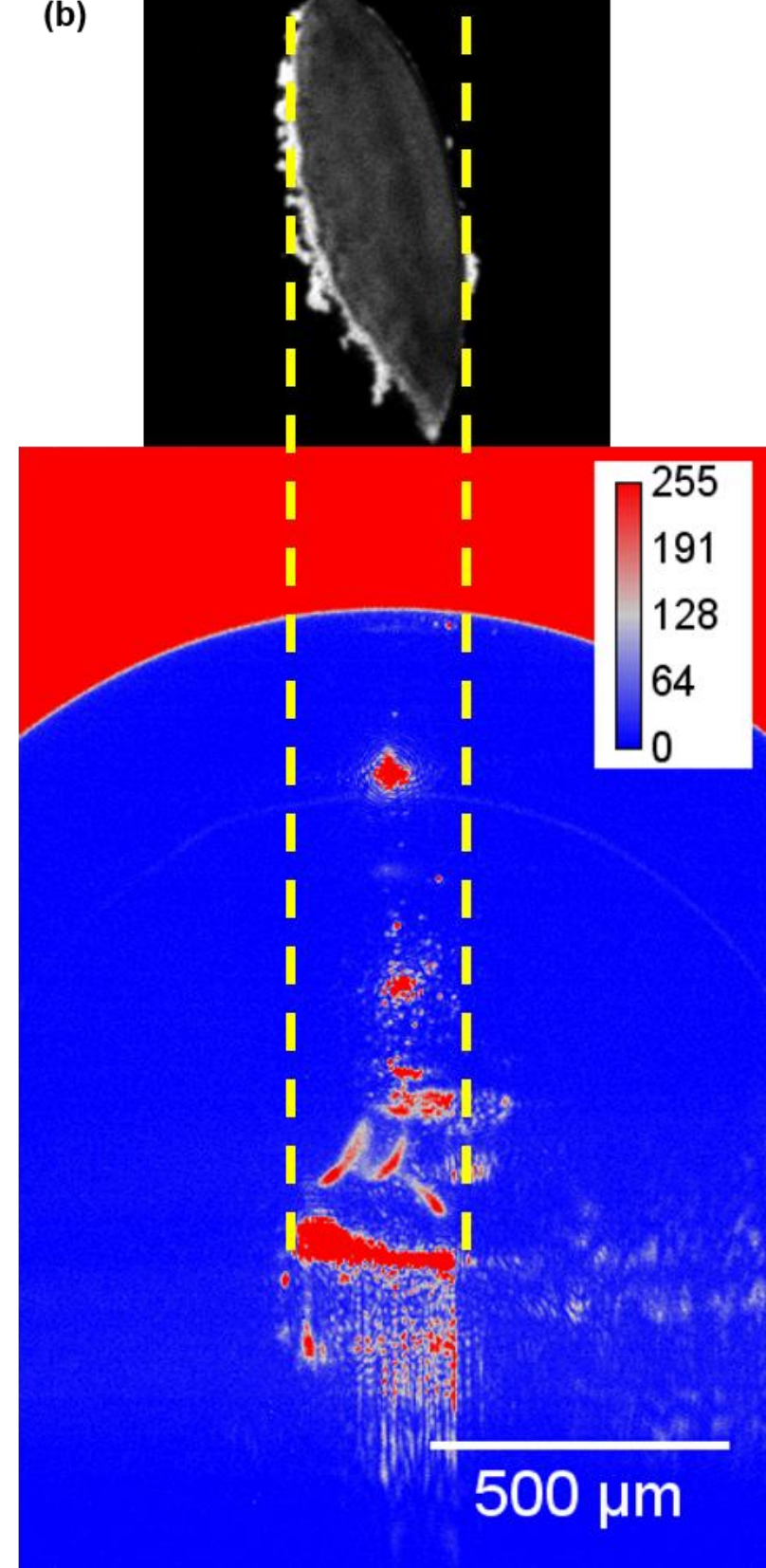

Figure S1. Correlation of the shape of ablation hole and laser scattering image for the cases of (a) round and (b) intentionally elongated ablation holes under the same pump pulse fluence. Both scattering images are processed by ImageJ for better visualization. As the vertical yellow dashed lines suggest the probe pulse illumination is more homogeneous through the round ablation hole, while the influence of the film rims which may have been created around the hole upon ablation is not visible. 


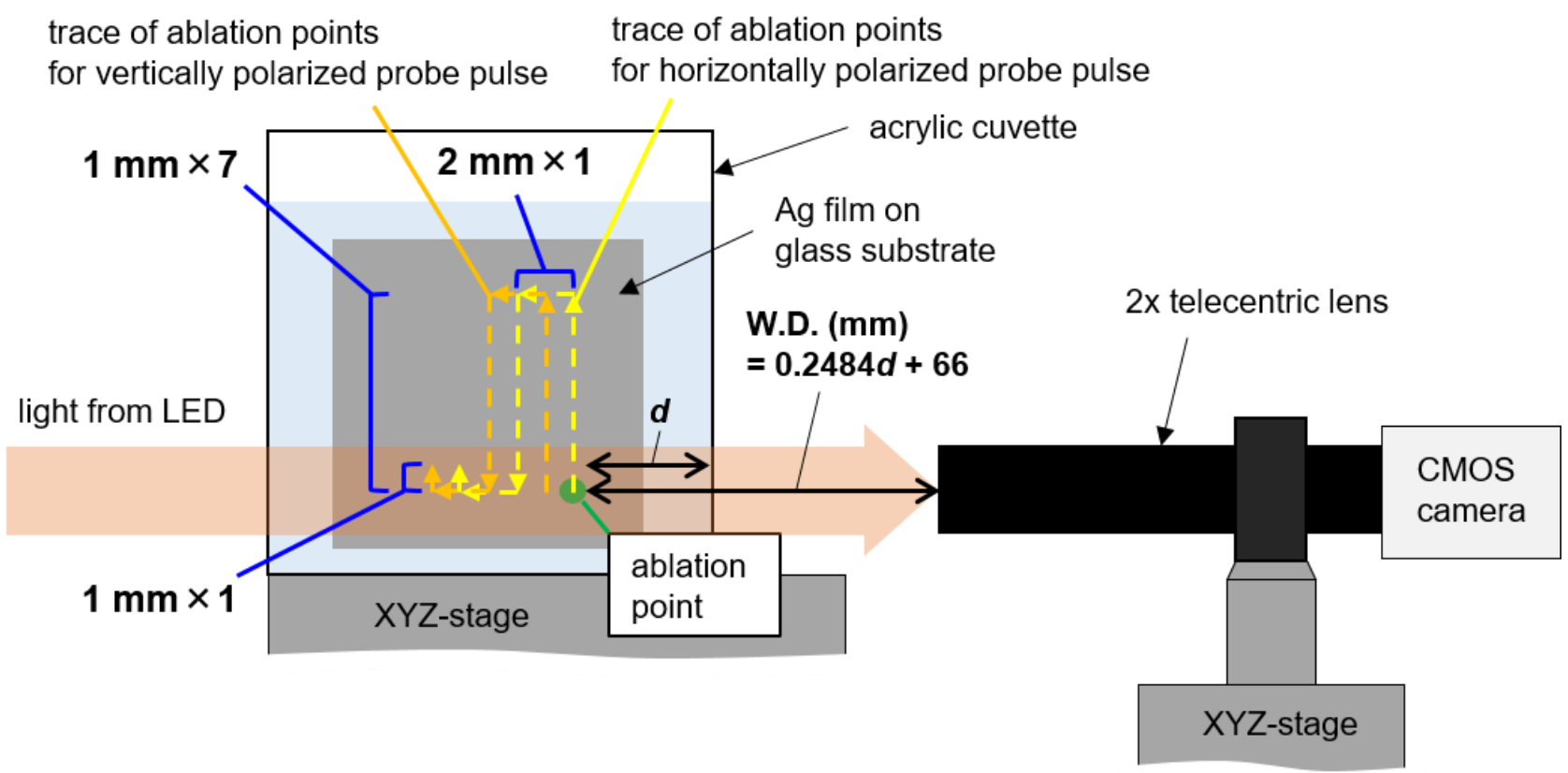

Figure S2. Side view of the acrylic cuvette $(50 \mathrm{~mm} \times 50 \mathrm{~mm} \times 50 \mathrm{~mm})$ on a motorized XYZ-stage where the pump pulse is horizontally (i.e., vertically-to-paper) incident onto the Ag film. After every measurement with a set of pump pulse_and time-delayed probe pulse together with the CMOS camera exposure, the cuvette and hence the Ag film is translated by $1 \mathrm{~mm}_{-}$with a stepping motor, as shown by the dashed yellow arrow. Then, water around the next ablation point is flushed away by small amount of pure water in a micropipette. Since the total amount of water in the cuvette is so much for the total number of pump pulses employed for the scattering experiments the above procedure works well to wash out the fragments as well as nanoparticles produced by the previous pump pulse from the new ablation point. The position of the CMOS camera is moved by $0.5 \mathrm{~mm}$ horizontally only when the acrylic cuvette is moved horizontally by 2 $\mathrm{mm}$ to correct the focal position of the objective lens. 


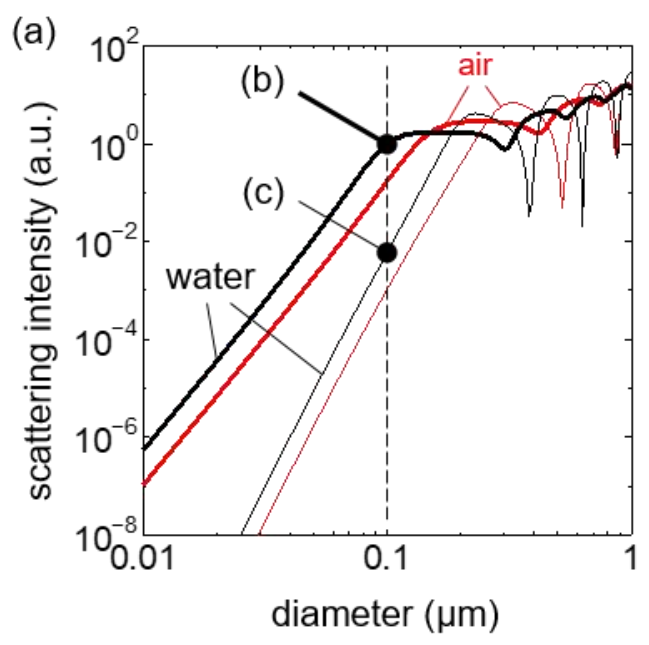

(b)

(c)

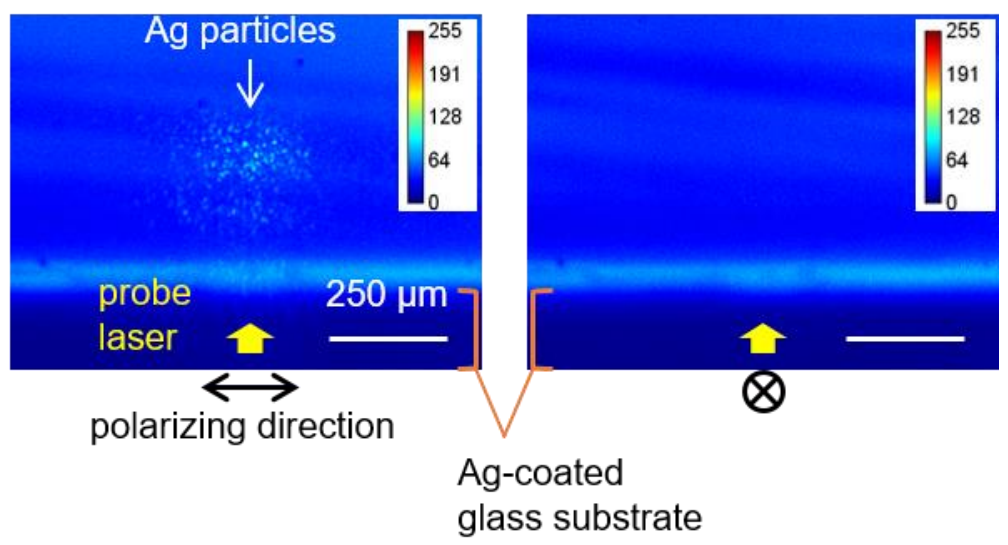

Figure S3. (a) Scattering intensities of Ag particles in water (black lines) and in air (red lines), respectively, as a function of Ag particle diameterat the scattering angle of 90 degrees. The polarization direction of the probe laser is assumed to be horizontal (thick lines) or vertical-to-paper (thin lines). All the intensities are normalized by that of Ag particles with a diameter of $100 \mathrm{~nm}$ in water for the horizontally polarized probe laser. (b) and (c) Laser scattering images of Ag particles with a diameter of $100 \mathrm{~nm}$ (Sigma Aldrich, lot number: 730777, $0.02 \mathrm{mg} / \mathrm{mL}$ ) in water. The brightness of the two images is doubled for better visibility. The $10 \mu \mathrm{L}$ colloidal solution of Ag particles is ejected just above a laser-created transparent window on an $\mathrm{Ag}$ film on a glass substrate which immersed in water purified by a syringe filter (mean pore size $0.22 \mu \mathrm{m}$, Membrane Solutions). Upon making the local number density of the colloidal solution sufficiently high only above the transparent window, we send the linearly polarized probe laser through the transparent window. The polarization direction of the probe laser is indicated by the arrow and cross marks in panels (b) and (c).

Through a separate measurement on the optical density (OD) at $532 \mathrm{~nm}$ we find that OD 0.8 for the same colloidal solution in a $1 \mathrm{~cm}$ cell, and this suggests that the scattering signals we see in (b) and (c) are in the single scattering regime. 


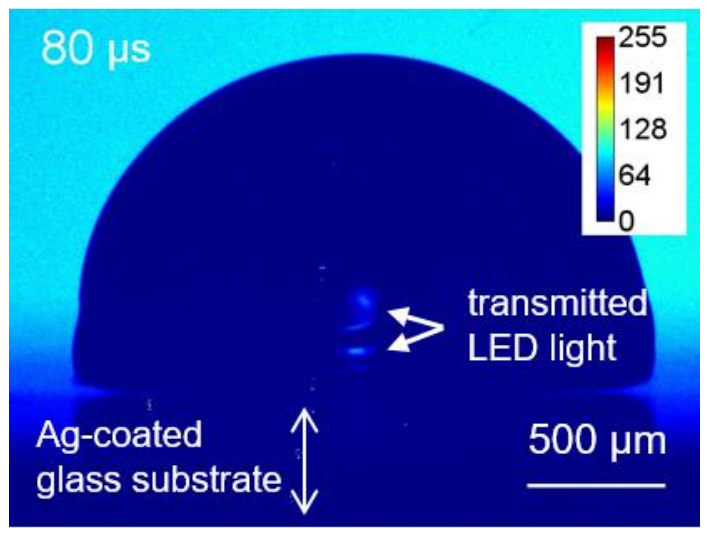

Figure S4. Shadowgraph image of the ablation bubble at $80 \mu$ s delay after the pump pulse where the bubble size is maximum and hence the curvature of the bubble surface is smallest. Note that the probe laser is off for this image. The dim blurred spots around the centre of the bubble originate from the LED light transmitted through the bubble. Note that they appear at the height where the normal of the bubble surface is parallel to the optical axis from the LED light to the camera. 
(a) O LED

$\times$ probe laser
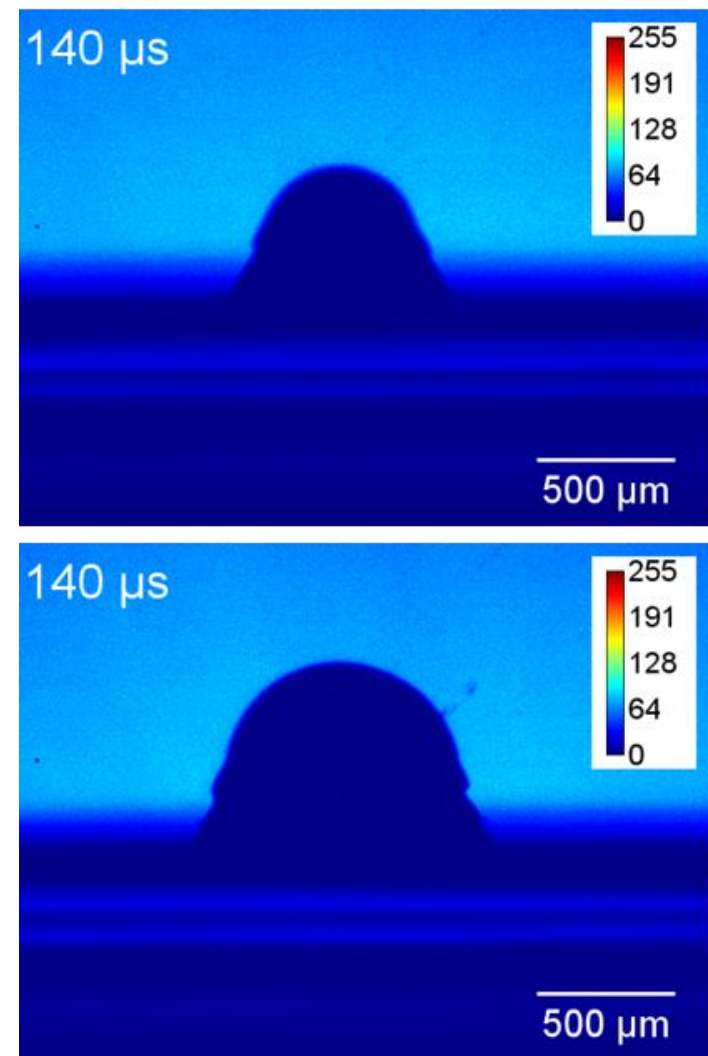

(b) $\times$ LED

O probe laser
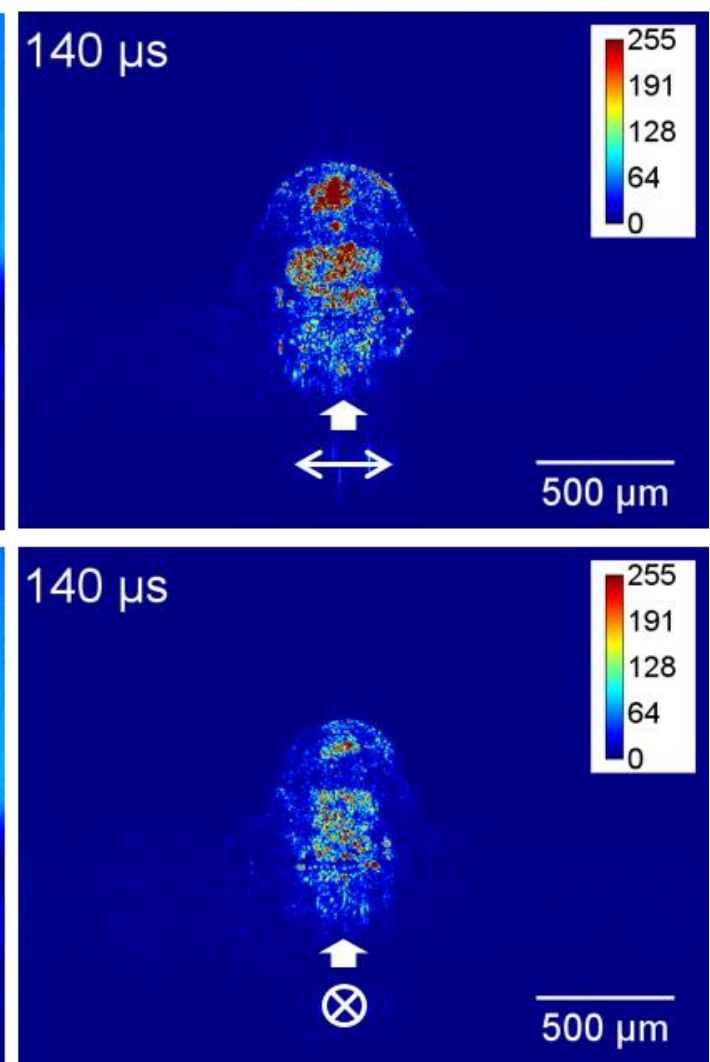

Figure S5. (a) Shadowgraph and (b) laser scattering images of the shrinking bubble taken at $140 \mu$ selay with the LED light and probe pulse only, respectively, to clarify the influence of LED light illumination on the laser scattering image. The dim and blurred spots which appear around the centre of the bubble at $80 \mu \mathrm{s}$ delay (Fig. S3) are not seen anymore because the diffraction of the LED light at the higher curvature of the shrinking bubble surface severely attenuates the transmission of the LED light toward the camera. 